\title{
Nonindependent and nonstationary response times in stopping and stepping saccade tasks
}

\author{
MatTheW J. Nelson \\ California Institute of Technology, Pasadena, California \\ AND \\ Leanne Boucher, Gordon D. Logan, Thomas J. Palmeri, and Jeffrey D. Schall \\ Vanderbilt University, Nashville, Tennessee
}

\begin{abstract}
Saccade stop signal and target step tasks are used to investigate the mechanisms of cognitive control. Performance of these tasks can be explained as the outcome of a race between stochastic go and stop processes. The race model analyses assume that response times (RTs) measured throughout an experimental session are independent samples from stationary stochastic processes. This article demonstrates that RTs are neither independent nor stationary for humans and monkeys performing saccade stopping and target-step tasks. We investigate the consequences that this has on analyses of these data. Nonindependent and nonstationary RTs artificially flatten inhibition functions and account for some of the systematic differences in RTs following different types of trials. However, nonindependent and nonstationary RTs do not bias the estimation of the stop signal RT. These results demonstrate the robustness of the race model to some aspects of nonindependence and nonstationarity and point to useful extensions of the model.
\end{abstract}

Cognitive control is revealed in experiments that require subjects to change their performance in response to changes in their environment (e.g., Logan, 1985). The stop signal task (Logan, 1994; Verbruggen \& Logan, 2008b) and the target step task (Camalier et al., 2007; Murthy, Ray, Shorter, Schall, \& Thompson, 2009) have been used to examine executive control of saccadic eye movements in humans and macaque monkeys (Camalier et al., 2007; Hanes \& Schall, 1995). These tasks present a target for an eye movement and then present either a stop signal, which indicates that the eye movement should be withheld, or a stepped target, which indicates that the eye movement should be directed to a new location. Performance on these tasks can be understood as the outcome of a race between a go process that makes the initial saccade and a stop process that inhibits the initial saccade to maintain fixation or to allow a new saccade to the new location (Camalier et al., 2007; Logan \& Cowan, 1984; see also Boucher, Palmeri, Logan, \& Schall, 2007). The race model assumes that the finish times for the go and stop processes as a function of trial number are stationary stochastic processes with independence between trials. This article reports data that challenge those assumptions and explores the consequences of those violations for analyses based on the race model. Our goal is not to evaluate the causes of nonindependence and nonstationarity but, rather, to document them in stopping and stepping tasks and evaluate their effects on race model and trial history analyses.
Nonstationarity refers to a stochastic process described by a mean or variance that changes over time. Response times (RTs) gradually becoming longer from the beginning to the end of an experimental session is one example of nonstationarity. Nonindependence refers to statistical dependence across samples in a time series. A correlation in RT between successive trials is one example of nonindependence. A time series that is nonstationary must be nonindependent, but the reverse is not necessarily true (e.g., autoregressive and moving average models; Wagenmakers, Farrell, \& Ratcliff, 2004).

The fact that RTs are often nonstationary and nonindependent is well established (e.g., Gilden, 2001; Wagenmakers et al., 2004). For instance, RT on a given trial can vary with the stimulus and response that occurred on the preceding trial (e.g., Fecteau \& Munoz, 2003; Luce, 1986). Furthermore, RT can change with arousal, fatigue, learning, and motivation throughout a session (Broadbent, 1971; Freeman, 1933; Welford, 1968, 1980). Several investigators have documented apparently systematic changes in RT during performance of the stop signal task (Cabel, Armstrong, Reingold, \& Munoz, 2000; Emeric et al., 2007; Kornylo, Dill, Saenz, \& Krauzlis, 2003; Li, Krystal, \& Mathalon, 2005; Özyurt, Colonius, \& Arndt, 2003; Rieger \& Gauggel, 1999; Schachar et al., 2004; Verbruggen \& Logan, 2008b; Verbruggen, Logan, Liefooghe, \& Vandierendonck, 2008). For example, the RT decreases after nostop-signal trials and increases after stop signal trials.

J. D. Schall, jeffrey.d.schall@vanderbilt.edu 
Race model analyses of stopping and stepping tasks focus on two measures of performance. First is the inhibition function, the probability of failing to cancel the response to the initial stimulus on a stop or step trial as a function of the interval between the onset of the initial stimulus and the stop or step signal (stop signal delay [SSD] or target step delay [TSD]). Second is the RT on trials with and without a stop (step) signal. From these quantities can be derived a measure of the time needed to interrupt the initial response. This measure is referred to as the stop signal RT (SSRT) in stopping tasks and the target step RT (TSRT) in stepping tasks.

Here, we first explore whether RT is nonindependent and nonstationary and how this impacts estimates of SSRT and TSRT derived from the race model. We also explore how nonindependence and nonstationarity might impact measures of trial-to-trial adaptations of RT. To address these issues, we measured the extent to which RTs were nonindependent and nonstationary across trials during performance of saccade stopping and stepping tasks by humans and macaque monkeys and assessed the impact of this on conventional analyses of these data.

\section{METHOD}

With the exception of the search step data from Monkey T, all the data presented here have formed the basis of previous publications (Boucher, Palmeri, et al., 2007; Boucher, Stuphorn, Logan, Schall, \& Palmeri, 2007; Camalier et al., 2007; Hanes, Patterson, \& Schall, 1998; Paré \& Hanes, 2003). Here, we reanalyze these data with a focus on the magnitude and impact of nonindependence and nonstationarity of RT.

\section{Stop Signal Task}

In the stop signal task, no-signal and stop signal trials were randomly interleaved (Figure 1). On no-signal trials, the subjects fixated a central point until it disappeared, whereupon a peripheral target appeared without any distractors. The subjects were then required to shift gaze to that location. On stop signal trials, the central fixation point reappeared following a variable delay after the appearance of the target. We refer to this variable delay as the SSD. On these trials, the subjects were instructed to cancel any impending saccade and maintain fixation on the initial fixation position. We refer to these trials as canceled trials (they are also called signal-inhibit trials in the literature). Monkeys were rewarded following both the canceled stop signal trials and correct no-signal trials. Because the occurrence and timing of the stop signal was unpredictable, on some trials, the subjects could not cancel their movement but, instead, made a sac-

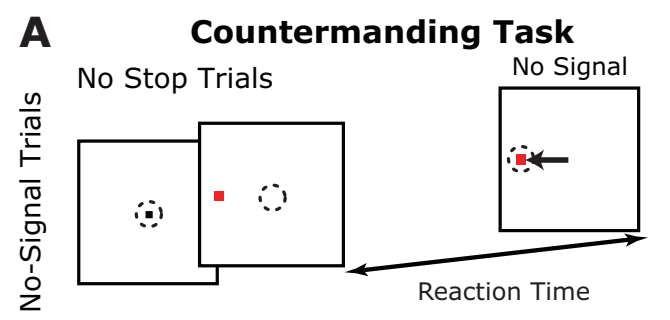

\section{B Search Step Task}

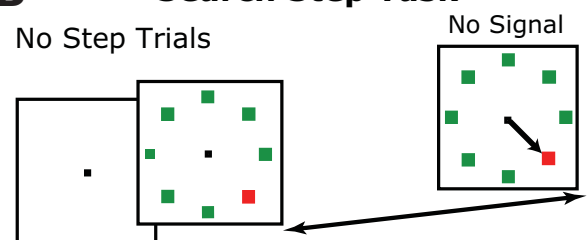

Reaction Time

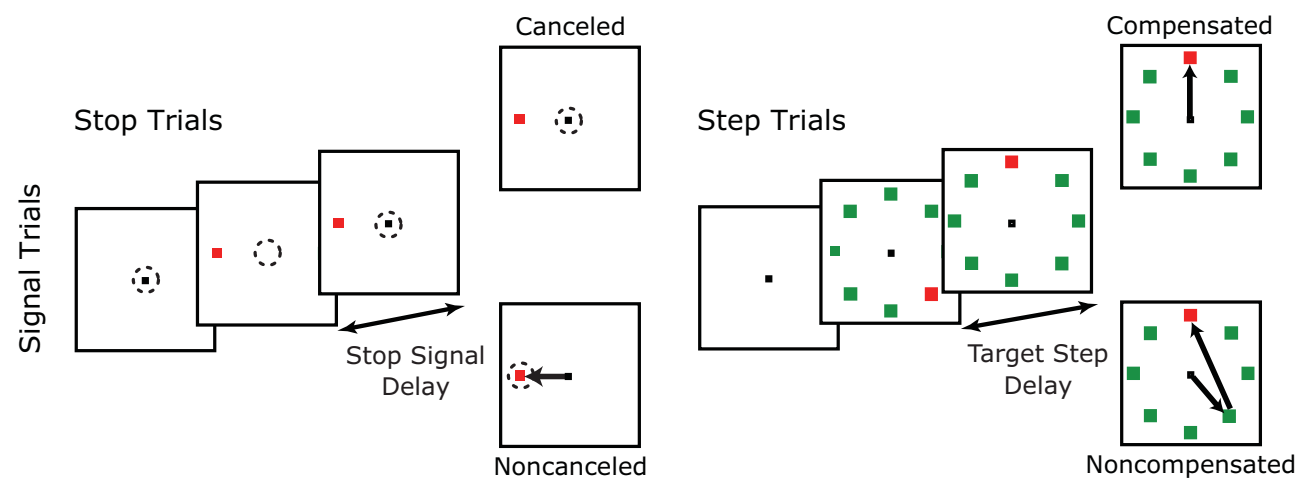

Figure 1. The experimental tasks. (A) Stopping. The dotted circle indicates the subject's gaze within each frame. A one-sided arrow indicates a saccade. A majority of the trials are no-signal trials, on which the subject fixates centrally and responds to a peripheral target that appears by making a saccade to it. A minority of the trials are stop trials, on which, following some delay after the presentation of the target, the central fixation point reappears, directing the subject to maintain central fixation. If the subject is able to successfully withhold the impending saccade, the trial is labeled as a canceled trial. If the subject errantly makes a saccade to the target, the trial is labeled as a noncanceled trial. (B) Search step. A one-sided arrow indicates a saccade the subject makes. A majority of the trials are no-signal trials, on which the subject fixates centrally and responds to an array of stimuli that appears by making a saccade to the oddball target. A minority of the trials are step trials, on which, following some delay after the presentation of the target, the location of the oddball singleton moves to a different location on the array through two isoluminant color changes, directing the subject to make a saccade to the new location of the target. If the subject is able to successfully make a saccade to the new target location, the trial is labeled as a compensated trial. If the subject errantly makes a saccade to the target, the trial is labeled as a noncompensated trial. 
cade to the target. We refer to these error trials as noncanceled trials (they are also called signal-respond trials in the literature). Monkeys were not rewarded following these trials.

In this article, we consider data collected for this task from 5 monkeys (Macaca mulatta and Macaca radiata) (Hanes et al., 1998; Paré \& Hanes, 2003) and 5 human subjects (Boucher, Stuphorn, et al., 2007). For the monkeys, the target could appear in one of two locations: either to the left or the right of the fixation point, positioned in the receptive field or movement field of a neuron.

For the human subjects, the target could appear in one of four locations: in the upper or lower left or in the upper or lower right, relative to the fixation point. The proportion of stop trials varied from $10 \%$ to $70 \%$ for monkeys (typically, $25 \%$ ) and was $30 \%$ for humans. The mean elapsed time between the start of consecutive trials was $\sim 4 \mathrm{sec}$ for monkeys and humans. Target eccentricities were $8.5^{\circ}$ for humans and varied between $4^{\circ}$ and $16^{\circ}$ for monkeys, according to receptive field location for the neuron recorded as part of the neurophysiological experiment. More task details are available in the cited publications.

\section{Search Step Task}

In the search step task, no-signal and target step trials were randomly interleaved (Figure 1). On no-signal trials, the subjects were required to shift gaze to a color singleton, either a red target among green distractors or a green target among red distractors. The color of the singleton varied across sessions. On target step trials, the target stepped to a different location in the array after a variable delay after its appearance in its initial location. We refer to this variable delay as the TSD. On these trials, the subjects were instructed to cancel their response to the initial location and shift their gaze directly to the new location (i.e., to compensate for the target step). We refer to these trials as compensated trials. Monkeys were rewarded following both compensated target step trials and correct no-signal trials. Because the occurrence, timing, and location of the steps were unpredictable, on some trials, the subjects could not compensate for the step but, instead, made a saccade to the initial target location. We refer to these error trials as noncompensated trials. Monkeys were not rewarded following these trials.

In this article, we consider data collected for this task from 4 monkeys (Murthy et al., 2007; Murthy et al., 2009) and 3 human subjects (Camalier et al., 2007). For the majority of the sessions in which the monkey data were collected, the target appeared with seven distractors (set size of eight). The target and distractors were evenly spaced in a circle around the central fixation point at the eccentricity of the receptive field of the neurons $\left(4^{\circ}-16^{\circ}\right)$. For the human data, one, three, or seven distractors appeared with the target randomly from trial to trial for most sessions at a fixed eccentricity of $9.5^{\circ}$. On a subset of sessions with monkeys and a subset of blocks within sessions with humans, the target appeared without any distractors. This condition is equivalent to the familiar double-step task (e.g., Becker \& Jürgens, 1979). For both humans and monkeys, the effects described here did not vary between the double-step and search step tasks with different set sizes, so we combined the data from the two tasks. The proportion of step trials varied from $25 \%$ to $50 \%$ for monkeys and was $40 \%$ for humans. The mean elapsed time between the start of consecutive trials was $\sim 3 \mathrm{sec}$ for monkeys and $\sim 5 \mathrm{sec}$ for humans.

\section{Manipulation of the SSD or the TSD}

In the stopping and stepping tasks, the SSD and TSD are independent variables. When the delay is short, subjects are more likely to cancel the impending saccade. When the delay is long, subjects are more likely to make a saccade to the initial target location. For some sessions presented here, the values of SSD (TSD) were predetermined and presented randomly throughout the session, independently of the subject's behavior. We refer to these as randomized SSDs (TSDs). In other sessions, a one-up/one-down staircase was used to adjust SSD (TSD) on each trial on the basis of the subject's behavior. In this procedure, the delay was increased by a predeter- mined amount (50 msec for humans in both tasks, $17-50 \mathrm{msec}$ for monkeys) following each canceled or compensated trial and was decreased by the same amount following each noncanceled or noncompensated trial. The goal of this procedure was to ensure that the subjects would respond successfully to the stop signal or target step on around $50 \%$ of the trials (Logan, Schachar, \& Tannock, 1997; Osman, Kornblum, \& Meyer, 1990). We refer to these as staircased SSDs (TSDs). Most of the data for the stopping task were recorded using randomized SSDs, with the exception of data from Monkeys $\mathrm{H}$ and $\mathrm{N}$, which were primarily recorded using a staircase procedure. All of the search step data were recorded using the staircase procedure. For certain analyses pertinent to the manner of SSD or TSD selection, subsets of data were grouped and analyzed on the basis of whether or not the staircase procedure was used.

Stop task SSDs ranged from 25 to $275 \mathrm{msec}$ for humans and from 25 to $450 \mathrm{msec}$ for monkeys for both staircased and nonstaircased data. Step task TSDs ranged from 50 to $250 \mathrm{msec}$ for humans and from 33 to $250 \mathrm{msec}$ for monkeys. Monitor refresh rates varied between subjects and tasks but were either 60 or $80 \mathrm{~Hz}$, depending on the experimental equipment used.

\section{Race Model Accounts of Performance in Stopping and Stepping}

Performance in the stop signal task can be understood as the outcome of a race between two stochastic processes, a go process and a stop process (Logan, 1994; Logan \& Cowan, 1984; see also Boucher, Palmeri, et al., 2007). The process that finishes first determines which behavior is produced. Recently, this model has been extended to the search step and double-step task with the addition of a second go process to produce the compensated saccade (Camalier et al., 2007; see also Verbruggen, Schneider, \& Logan, 2008). The race model formulation affords the ability to calculate the correct time needed to interrupt preparation of the initial movement (Logan \& Cowan, 1984). This time is referred to as the SSRT for the stop signal task and the TSRT for the search step task.

We used two methods to estimate SSRT and TSRT (Figure 2). With the integration method, SSRT or TSRT can be calculated for each SSD or TSD by integrating the no-signal RT distribution until the proportion of RTs is equal to the proportion of noncanceled or noncompensated trials on the inhibition or compensation function for a particular SSD or TSD. The SSRT or TSRT is then given by that point in time minus the SSD or TSD. Using this method, an estimate of SSRT or TSRT is determined for each SSD or TSD, with the overall measure typically averaged across SSDS or TSDs. With the difference method, the mean of the inhibition (compensation) function is calculated by treating the function as a cumulative distribution function. The SSRT or TSRT is then equal to the mean of the no-signal RT distribution minus this value. The SSRTs and TSRTs we report in this article are the average of the values from these two methods.

\section{RT Spectra}

Power spectra quantify trial-by-trial nonindependence in RT data (Gilden, 2001; Wagenmakers et al., 2004). If the processes producing RTs in a time series are independent across trials (which also requires stationarity), the spectrum of the data series will be flat, like the spectrum of white noise sampled with the same frequency. To test whether RT data deviate from this prediction, we estimated the power spectra of RT series and averaged them across sessions for each subject. Power spectra of RTs on no-signal trials were estimated using the Lomb-Scargle method for unevenly sampled data (Lomb, 1976; Scargle, 1982), with stop or step trials treated as missing data. For each session, this produced a spectral estimate with frequency step sizes equal to the reciprocal of the number of trials in the session. The resulting frequencies ranged from one such step up to the Nyquist limit. Adjacent frequency components were averaged into bins spaced evenly on a logarithmic scale and then were averaged across each session to give a spectral estimate for each subject. Confidence intervals were calculated using the assumption that the spec- 

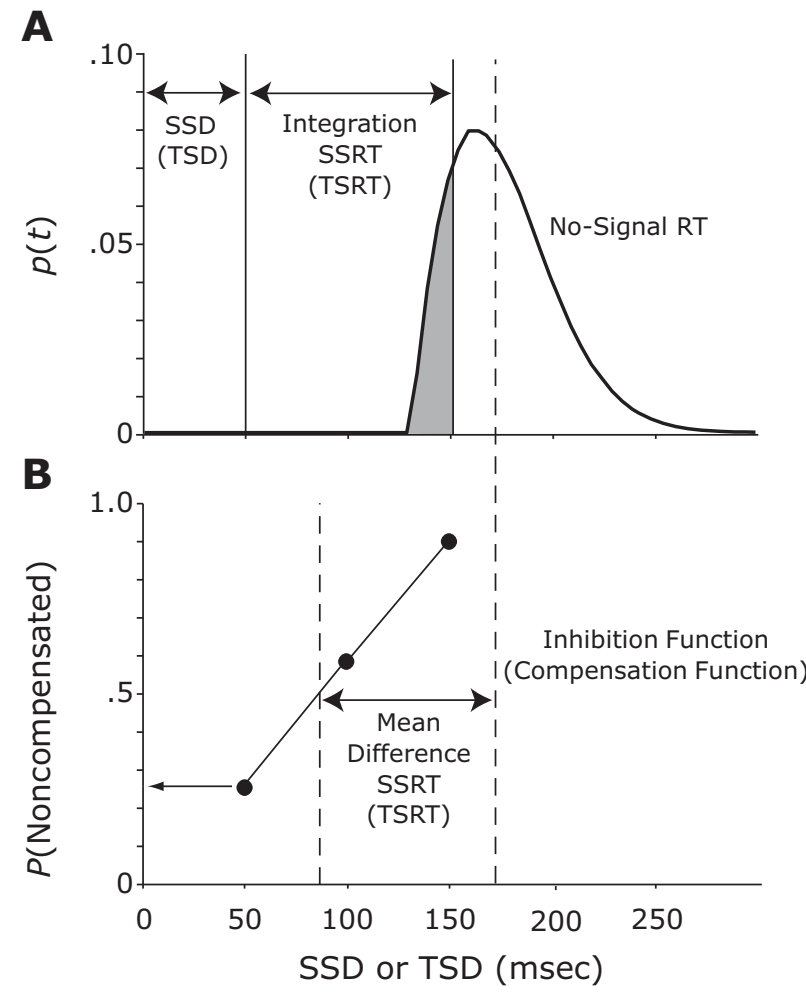

Figure 2. Illustration of how the stop signal response time (SSRT) and target step response time (TSRT) are calculated according to the race model. (A) Probability density of RTs on trials with no stop signal. Mean of the distribution is indicated by the vertical dashed line. Duration of the stop signal delay (SSD; target step delay [TSD]) and of SSRT (TSRT) is indicated by horizontal arrows. The shaded portion of the function indicates the proportion of trials on which a response would have occurred before the critical time of SSD + SSRT (TSD + TSRT) for a given SSD (TSD). (B) Inhibition function plotting the probability of responding on trials calling for a stop (step) response as a function of SSD (TSD). The lower horizontal arrow highlights the fraction of failures for the shortest SSD (TSD), which corresponds to the shaded area in panel $A$. The SSRT (TSRT) is determined from the difference between the mean RT on no-signal trials and the midpoint of the inhibition function, indicated by the upper horizontal arrow.

tral density estimates follow a chi-square distribution with degrees of freedom equal to twice the number of averaged estimates (Jarvis $\&$ Mitra, 2001). We considered this to be the number of sessions for each subject. For comparison, we randomly permuted the trial order once for each session and estimated the trial-shuffled spectrum by the same procedure as that used for the original data. For more details on these methods, please see Appendix A.

\section{RESULTS}

Data were obtained from humans and macaque monkeys performing a stop signal task and a search step task with saccadic eye movements. All statistical tests were performed at a .05 significance level.

\section{Nonindependent and Nonstationary RTs}

We observed fluctuations in RT during the course of a session occurring on immediate (1 trial), local ( 10100 trials), and global ( 1,000 trials) time scales. Fluctua- tions on all of these scales can be seen in Figure 3, which shows a representative session of a monkey performing the step task. The raw RTs are presented in the top panel, and the 100-trial running averages of RT, TSD, and percentage of step trials are shown in the middle and lower panels. Immediate variability can be attributed to the irreducible randomness of RT or adaptive control across successive trials. Local variation of RT often coincided with gradual changes of the running fraction of step trials or average TSD (see also Emeric et al., 2007). Global variation could be expressed as a gradual slowing (or speeding) of RT across a session, probably resulting from extraneous factors not controlled or manipulated by the experiment, such as fatigue or motivation fluctuations.

A gradual increase of RT mean and variance is evident in Figure 3. To assess this for all of the sessions, we divided each session into thirds by trial number and compared the mean and variance between the initial and final thirds of each session. Table 1 summarizes the trends, indicating the numbers and percentages of sessions with significant increases or decreases of RT mean and variance (ranksum for mean, Levene's nonparametric test for variance, both two-tailed; the expected chance level is $2.5 \%$ for each cell in the table). More sessions than would be expected by chance had significant changes in RT mean and variance, although across subjects and sessions, both decreases and increases of RT mean and variance were observed. Thus, RTs were nonstationary.

To quantify the degree of independence of RTs across trials, we calculated the frequency spectrum of RTs for each subject. Figure 4 shows the power spectra for the successive RTs produced by each subject, with confidence intervals compared with the power spectra derived from a shuffled sequence of the same RTs. For both humans and monkeys in both the stepping and stopping tasks, spectral power was elevated at low frequencies. Across subjects, the power at the lowest frequencies was at least twice the power at the highest frequencies. We observed the same pattern when calculating the RT spectra after removing any linear trends from the data. Thus, RTs show that significant slow fluctuations beyond a linear trend occur during performance of these tasks. The higher power at low trial frequencies indicates that RTs within immediate and local time scales are expected to be positively correlated. We verified that this was the case for pairs of consecutive no-signal trials.

\section{Impact of Nonindependent and Nonstationary RTs on Inhibition Functions}

Performance in stopping or stepping tasks is characterized by the probability of failing to cancel the initial movement as a function of the delay of the stop or step signal. The relationship of this inhibition (or compensation) function to the distribution of RTs is used to calculate SSRT (or TSRT). Having already demonstrated the nonindependence and nonstationarity of RT, in this section, we consider the impact these have on the form of the inhibition (or compensation) function.

We plotted inhibition functions from different chronological epochs within sessions. Figure 5 displays data from 

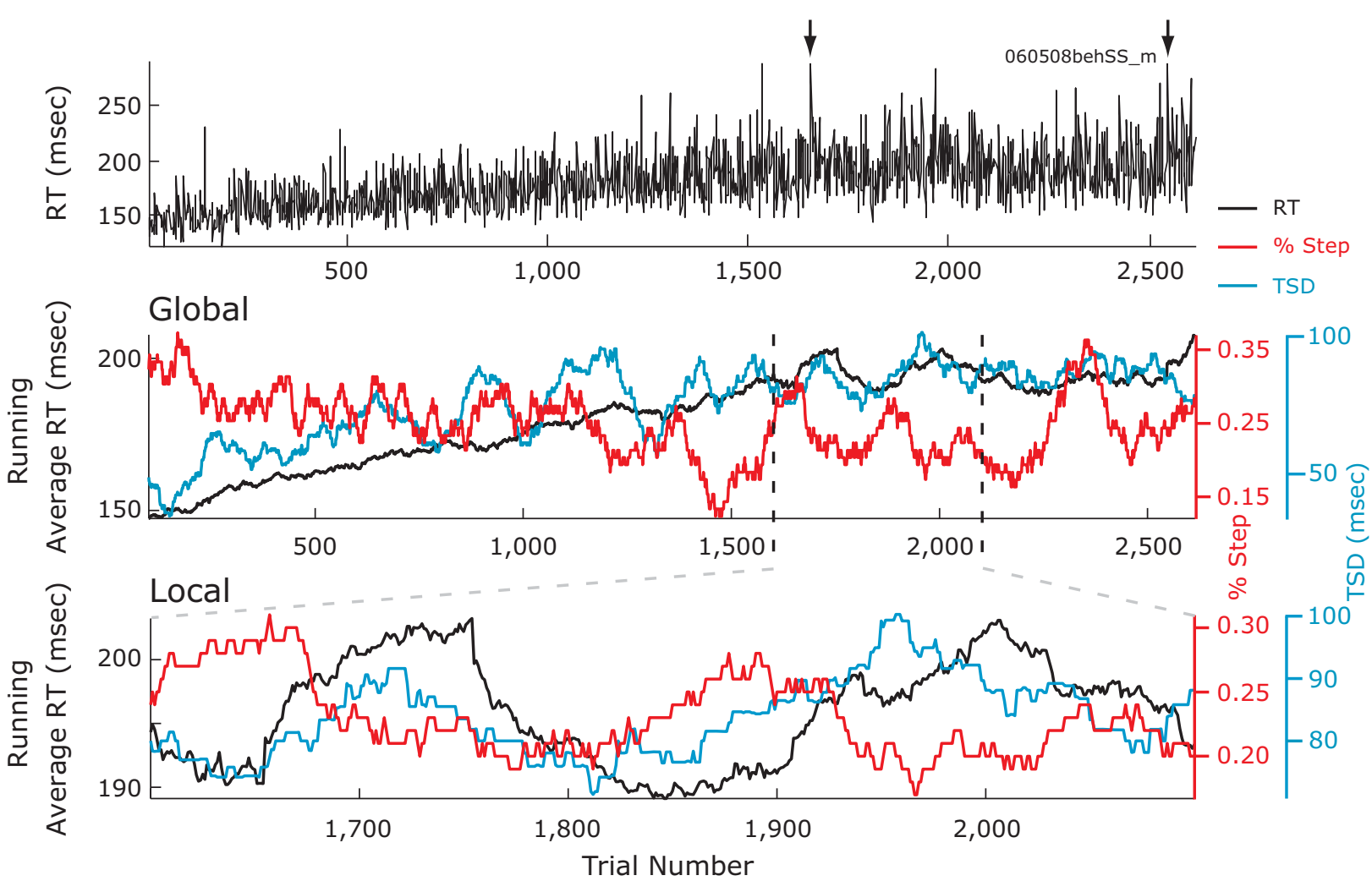

Figure 3. Immediate, local, and global variations of response time (RT), target step delay (TSD), and the percentage of step trials in a sample session of a monkey performing the search step task, with TSDs determined by a one-up/one-down staircase procedure. The top plot shows unfiltered no-signal RTs. Arrows indicate two trials with RTs that exceeded the scale of the plot (left, 431 msec; right, $556 \mathrm{msec}$ ). The middle plot shows 100-trial running average of the RT (black), TSD (blue), and percentage of step trials (red) for the same set of trials. The lower plot shows the same for a portion of the session.

a representative session in which a monkey performed the stop signal task with the SSD adjusted through the staircase procedure. Figure 5A shows that RT increased gradually over the course of the session, coincident with a gradual increase in SSD. Figure 5B compares the inhibition function derived over the entire session with inhibition functions derived from an early epoch (during which RT and SSD were shorter) and a later epoch (during which RT and SSD were longer). The inhibition function over the entire session had a shallower slope than did the inhibition functions from either epoch. Many sessions across species and tasks showed similar differences between session-level and epoch-level inhibition functions or compensation functions.

To compare inhibition functions across sessions, it has been shown previously that the abscissa of the inhibition function can be transformed to represent the relative finishing time of the stop and go processes by subtracting SSD and SSRT from the mean RT for each epoch (Logan \& Cowan, 1984). The logic of this transformation follows from the race model claim that the probability of inhibiting depends on the relative finishing time of these processes, not on the absolute finishing time of either process alone. Specifically, as is illustrated in Figure 5C, we produced a transformed SSD (referred to as SSD') by subtracting the difference between the mean RT in each epoch and the mean RT over the entire session from the original SSD:

$$
\mathrm{SSD}^{\prime}=\mathrm{SSD}-(\overline{\text { Epoch RT}}-\overline{\mathrm{RT}}) .
$$

This translates the inhibition functions from an epoch when a subject responds more quickly to higher values of $\mathrm{SSD}^{\prime}$ and translates the inhibition function from an epoch when a subject responds more slowly to lower values of $\mathrm{SSD}^{\prime}$. The transformed inhibition functions overlap each other as well as the inhibition function from the entire session. Note that this procedure does not change the slopes. We extended this epoch-by-epoch transformation procedure to a trial-by-trail transformation according to

$$
\mathrm{SSD}_{i}^{\prime}=\mathrm{SSD}_{i}-\left(\text { local } \mathrm{RT}_{i}-\overline{\mathrm{RT}}\right) .
$$

The $\mathrm{SSD}_{i}\left(\mathrm{TSD}_{i}\right)$ for the $i$ th stop (step) trial was transformed to $\mathrm{SSD}_{i}^{\prime}$ (or $\mathrm{TSD}_{i}^{\prime}$ ) by subtracting the difference between the average RT on no-signal trials in the 101-trial interval centered on that trial (represented by local $\mathrm{RT}_{i}$ above) and the session average RT ( $\overline{\mathrm{RT}}$ above $)$. The length of the averaging window was truncated as necessary near the start or end of a session. Resulting SSD' (TSD') values were binned, and the proportion of stop (step) trials on which the subject failed to cancel the initial responses in each bin was determined. The transformed inhibition (compensation) functions, which plot this proportion 
Table 1

Numbers and Proportions of Sessions for Each Subject With Significant Increases and Decreases to the Mean and Standard Deviation of Response Time When the First and Last of Three Evenly Sized Epochs in a Session Were Compared

\begin{tabular}{llcll}
\hline Subject & \multicolumn{1}{c}{$\begin{array}{c}\text { Mean } \\
\text { Increase }\end{array}$} & \multicolumn{1}{c}{$\begin{array}{c}\text { Mean } \\
\text { Decrease }\end{array}$} & $\begin{array}{c}\text { Standard } \\
\text { Deviation } \\
\text { Increase }\end{array}$ & $\begin{array}{c}\text { Standard } \\
\text { Deviation } \\
\text { Decrease }\end{array}$ \\
\hline \multicolumn{5}{c}{ Human Stop } \\
S.N. & $1 / 10(10 \%)$ & $1 / 10(10 \%)$ & $0 / 10(0 \%)$ & $1 / 10(10 \%)$ \\
J.B. & $2 / 6(33 \%)$ & $0 / 6(0 \%)$ & $0 / 6(0 \%)$ & $0 / 6(0 \%)$ \\
K.W. & $3 / 9(33 \%)$ & $1 / 9(11 \%)$ & $0 / 9(0 \%)$ & $0 / 9(0 \%)$ \\
E.F. & $2 / 9(22 \%)$ & $1 / 9(11 \%)$ & $0 / 9(0 \%)$ & $0 / 9(0 \%)$ \\
E.L. & $1 / 7(14 \%)$ & $1 / 7(14 \%)$ & $1 / 7(14 \%)$ & $0 / 7(0 \%)$ \\
\multicolumn{5}{c}{ Human Step } \\
C.C. & $9 / 38(24 \%)$ & $24 / 38(63 \%)$ & $2 / 38(5 \%)$ & $5 / 38(13 \%)$ \\
L.B. & $5 / 40(13 \%)$ & $16 / 40(40 \%)$ & $0 / 40(0 \%)$ & $3 / 40(8 \%)$ \\
S.S. & $14 / 40(35 \%)$ & $15 / 40(38 \%)$ & $4 / 40(10 \%)$ & $2 / 40(5 \%)$ \\
\multicolumn{5}{c}{ Monkey Stop } \\
A & $4 / 89(4 \%)$ & $3 / 89(3 \%)$ & $10 / 89(11 \%)$ & $3 / 89(3 \%)$ \\
C & $2 / 17(12 \%)$ & $2 / 17(12 \%)$ & $1 / 17(6 \%)$ & $2 / 17(12 \%)$ \\
F & $1 / 24(4 \%)$ & $3 / 24(13 \%)$ & $2 / 24(8 \%)$ & $1 / 24(4 \%)$ \\
H & $8 / 67(12 \%)$ & $6 / 67(9 \%)$ & $3 / 67(4 \%)$ & $1 / 67(1 \%)$ \\
N & $50 / 269(19 \%)$ & $45 / 269(17 \%)$ & $30 / 269(11 \%)$ & $21 / 269(8 \%)$ \\
\multicolumn{5}{c}{ Monkey Step } \\
C & $12 / 34(35 \%)$ & $2 / 34(6 \%)$ & $12 / 34(35 \%)$ & $1 / 34(3 \%)$ \\
F & $13 / 41(32 \%)$ & $8 / 41(20 \%)$ & $4 / 41(10 \%)$ & $5 / 41(12 \%)$ \\
L & $21 / 47(45 \%)$ & $3 / 47(6 \%)$ & $9 / 47(19 \%)$ & $4 / 47(9 \%)$ \\
T & $42 / 42(100 \%)$ & $0 / 42(0 \%)$ & $28 / 42(67 \%)$ & $1 / 42(2 \%)$ \\
\hline
\end{tabular}

Note-Means were tested using a nonparametric rank sum test, and variances were tested using Levene's nonparametric test. Both tests were two-tailed. The expected chance level is $2.5 \%$ for each cell in the table.

against the $\mathrm{SSD}^{\prime}\left(\mathrm{TSD}^{\prime}\right)$ values, allow us to probe the impact of nonindependence and nonstationarity on inhibition functions by providing an example of what the data would look like if fluctuations of mean RT were removed. The forms of these transformed inhibition (compensation) functions were characterized by cumulative Weibull function fits to the values. The slopes of the transformed inhibition (compensation) function were tested statistically as follows. We performed a permutation test comparing the median slopes of the transformed inhibition function with the slopes when trial order was randomly permuted 1,000 times before applying the transformation procedure.

The left panel of Figure 5D shows the fitted cumulative Weibull distributions for the original and transformed inhibition functions for this sample session. The number and proportion of sessions in which the transformed inhibition function was significantly steeper than the original inhibition function is shown in the first column of Table 2. Across the population, the transformed inhibition function is significantly steeper in most sessions. In the human search step data, this effect was less prevalent.

The foregoing analyses focused on data from sessions in which SSD (TSD) was adjusted on a staircase according to the subjects' performance. With the staircase procedure, a different range of SSD (TSD) values is presented within different epochs of an experimental session due to differences in RTs across epochs. To determine whether the underestimation of the inhibition function slope is specific to the use of staircasing, we performed the same analyses on data from a monkey performing the stop signal task with a set of SSDs randomly presented with equal probability. Figure 6A shows a 100-trial running mean of the RTs and the probability of not canceling on stop trials, with two epochs highlighted. Clearly, the probability of not canceling varied inversely with RT. For the same SSD values, subjects are more likely to inhibit the initial movement during an epoch of slower responding.

Figure 6B compares the inhibition functions from each epoch with that from the entire session. We compared the heights and slopes of these inhibition functions. The inhibition function from the epoch when the subject was responding more quickly lies above that from the epoch when the subject was responding more slowly. The inhibition function slopes between these particular epochs and the overall session were not noticeably different. However, when the same transformation as that applied to staircased sessions was applied to this session, the transformed inhibition function was again found to be significantly steeper than the original. This procedure was applied to each session with a fixed SSD (TSD). The number and proportion of sessions of nonstaircased data for each subject in which the transformed inhibition function was significantly steeper than the original is shown in the first column of Table 3. Across the population, this was significant for many nonstaircased sessions. Thus, a shallower slope of the inhibition function from an entire session seems to be a general consequence of nonindependence and is not due to adapting SSD (TSD) to performance via staircasing.

\section{Estimating SSRT or TSRT}

The transformation procedure was also used to calculate

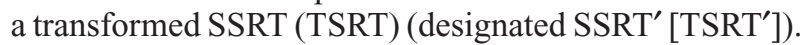
To do so, RTs were transformed as follows:

$$
\mathrm{RT}_{i}^{\prime}=\mathrm{RT}_{i}-\left(\text { local } \mathrm{RT}_{i}-\overline{\mathrm{RT}}\right) .
$$

The $\mathrm{RT}_{i}$ for the $i$ th trial with no stop (step) signal was transformed to $\mathrm{RT}_{i}^{\prime}$ by subtracting the difference between the local average RT and the session average RT as described above.

Naturally, the distribution of $\mathrm{RT}^{\prime}$ is centered on the session mean, but with less variance, as shown in the right panel of Figure 5D. Although modest, the significance of the reduction of variance for this session was verified statistically using another 1,000-shuffle permutation test comparing the standard deviation of the $\mathrm{RT}^{\prime}$ distribution. For the session illustrated in Figure 6, the reduction of no-signal RT variance by the transformation approached significance $(p=.055)$. For each subject, the number and proportion of sessions in which the transformation significantly reduced the RT standard deviation are shown in the second columns of Tables 1 and 2 for staircased and nonstaircased data, respectively. Adjusting for RT nonindependence and nonstationarity by this transformation procedure significantly reduced the variance of no-signal RT distributions in many sessions for all the subjects.

Using the $\mathrm{SSD}^{\prime}\left(\mathrm{TSD}^{\prime}\right.$ ) and $\mathrm{RT}^{\prime}$ values from which slow fluctuations in RT were removed, we obtained a transformed SSRT' (TSRT'). SSRT' and SSRT values were not significantly different for the session illustrated in Figure 5 
Human
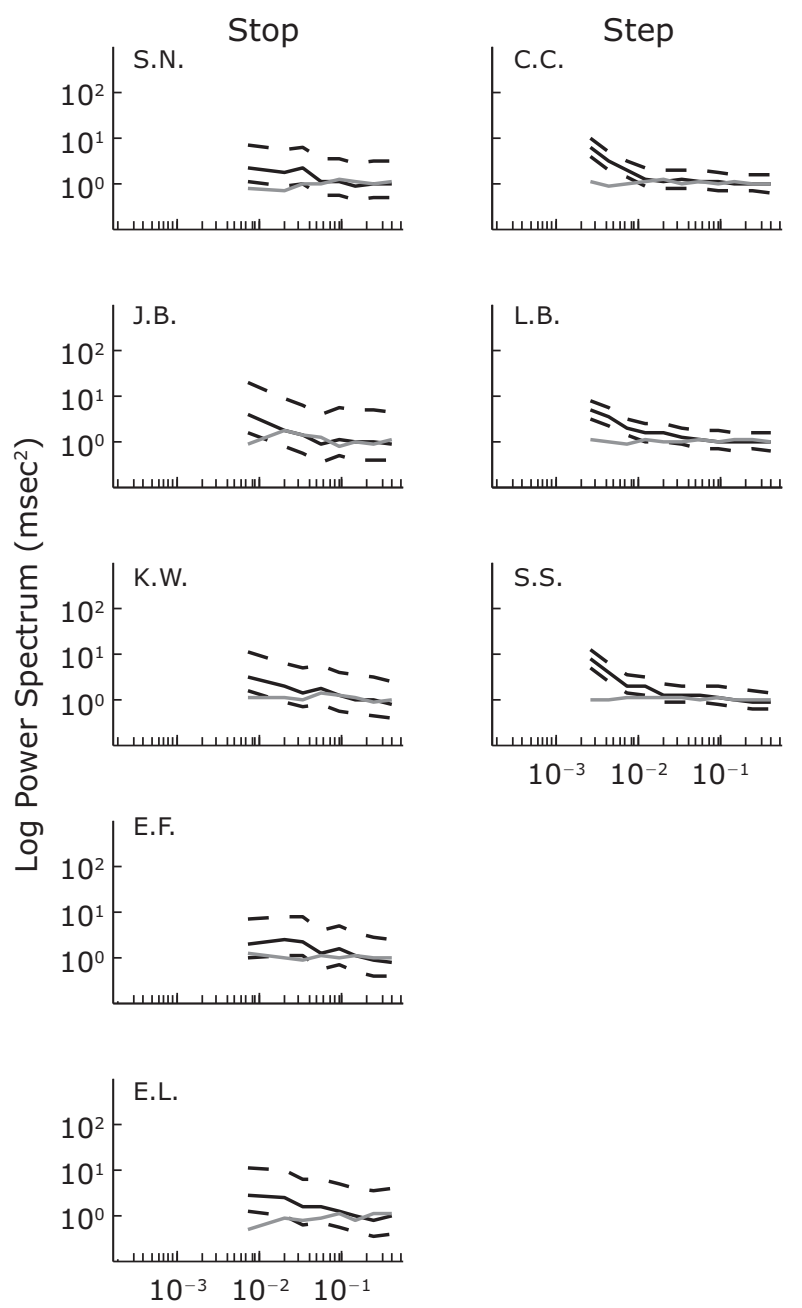

Monkey
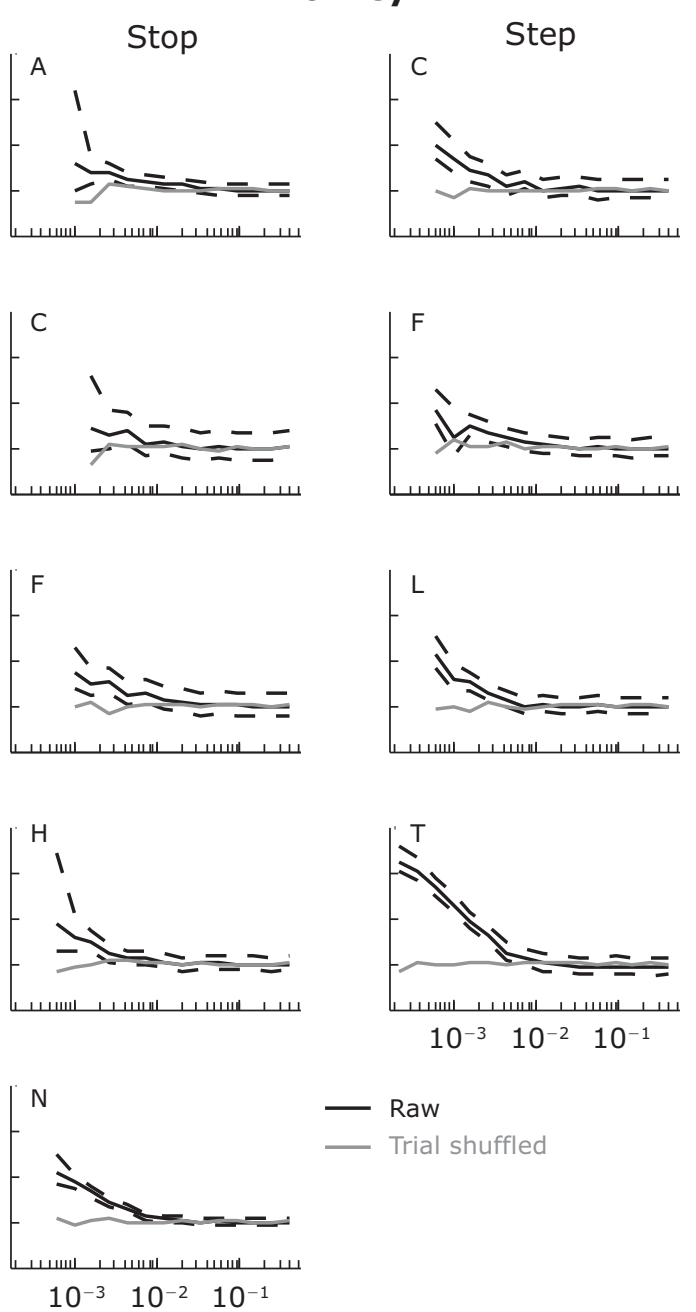

Log Cycles/Trial

Figure 4. Spectrum of response times (RTs). The panels show RT mean-square power spectra plotted against frequency in units of cycles per trial, averaged across sessions for each subject. For each panel, the solid black line shows the power spectrum, with $95 \%$ confidence intervals shown in the dashed lines. The gray line in each panel shows the spectral estimate of the same data when the trial order within each session is randomly permuted. Plots are collected in each column on the basis of the species and task for each subject, as indicated. Across species and tasks, RT spectra are significantly not flat for most subjects, with increased power at low frequencies and decreased power at high frequencies, as compared with that of the independent trial-shuffled spectrum.

$\left(\mathrm{SSRT}=141 \mathrm{msec}, \mathrm{SSRT}^{\prime}=136 \mathrm{msec}\right.$, permutation test $)$ or Figure $6\left(\mathrm{SSRT}=153 \mathrm{msec}, \mathrm{SSRT}^{\prime}=152 \mathrm{msec}\right.$, permutation test). The distributions of differences of SSRT' (TSRT') and SSRT (TSRT) across subjects and conditions are illustrated in Figure 7. Overall, accounting for the nonindependence and nonstationarity of RT had very little effect on the estimate of stop process duration SSRT (TSRT). The third columns of Tables 1 and 2 display for each subject the numbers and proportions of sessions in which SSRT' (TSRT') was significantly different from SSRT (TSRT). Adjusting for RT nonindependence and nonstationarity by this transformation rarely changed significantly the SSRT (TSRT). The only exception was Monkey T performing the search step task, for which 14 of 42 sessions showed a significant difference between TSRT and TSRT'.

Furthermore, we found no systematic bias in the distributions of SSRT' (TSRT') relative to SSRT (TSRT) (paired $t$ tests). Only Subject C.C. performing the step task exhibited a significant difference between TSRT' and TSRT [12.5 msec slower; $t(37)=3.3, p<.05]$. In spite of these idiosyncrasies, we conclude that the estimation of SSRT (TSRT) is robust against nonstationarity or nonindependence of RT. Moreover, in Appendix B we show mathematically that with fluctuating go and stop process finish time distributions, one method of measuring SSRT (TSRT) provides a time-weighted average of the SSRT (TSRT) value over the entire session. This suggests that 


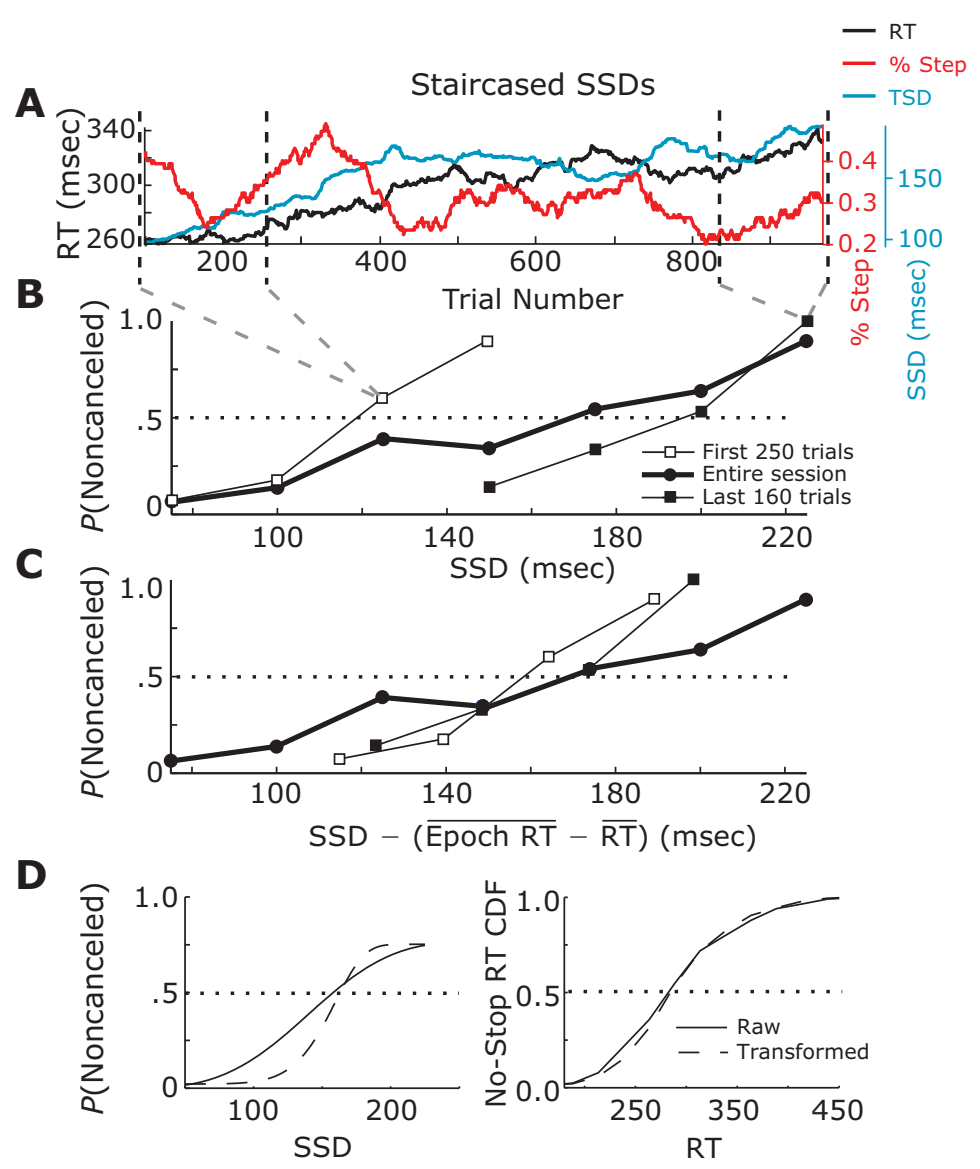

Figure 5. Effects of nonindependent and nonstationary response times (RTs) on inhibition functions for staircased data. (A) 100-trial running average of the RT (black), stop signal delay (SSD; blue), and percentage of step trials (red) for a sample session of a monkey performing the stopping task. SSDs for this session were determined by a one-up/one-down staircase procedure. (B) Comparison of inhibition function for an entire session with inhibition functions within two epochs. (C) Transformation of inhibition functions by subtracting from the SSD the difference between the mean no-signal RT in an epoch and the mean no-signal RT over the entire session. (D) Transformation of the inhibition function and of the no-signal RT distribution resulting from subtracting from each SSD and RT the difference between the mean local RT and the mean overall RT. Left panel shows original and transformed inhibition functions. Right panel shows original and transformed no-signal RT distributions. The stop signal RTs calculated from the original and transformed distributions were 141 and 136 msec, respectively.

even as a subject's RT fluctuates, the value of SSRT remains stable.

\section{Trial-by-Trial RT Adjustments During Stopping and Stepping}

A hallmark of executive control is the ability to change behavior on the basis of past stimuli, responses, and outcomes. Many studies have shown that subjects adjust their $\mathrm{RT}$ on the basis of the previous trial's stimulus parameters (e.g., Verbruggen \& Logan, 2008a) or their behavior on the previous trial (e.g., Emeric et al., 2007). These and related studies implicitly assume independence and stationarity in RT when they examine RTs on trial $n+1$ based on the stimuli or responses on trial $n$. However, if the occurrence of the stop (step) response on trial $n$ varies with fluctua- tions of RT alone, conclusions drawn from an examination of RTs on trial $n+1$ alone may be misleading.

A change in RT on trial $n+1$ could be due to an executive control signal influencing behavior on the basis of the outcome of trial $n$. Alternatively, relationships between performance on trials $n$ and $n+1$ could simply be consequence of nonindependence and nonstationarity of RT. Hence, a change in RT on trial $n+1$ may not be due to an executive control signal. One way to account for the effects of nonindependence and nonstationarity is to consider what happened on the $n-1$ trial.

Figure 8 shows the RTs on no-signal trials before $(n-1)$ and after $(n+1)$ all three trial types for the stop task (no signal, canceled, and noncanceled) and the step task (no signal, compensated, and noncompensated). Colors for 
Table 2

Numbers and Proportions of Staircased Sessions for Each Subject in Which a Transformation to Remove Response Time (RT) Fluctuations Significantly Affected Indicated Values at a $p<.05$ Level

\begin{tabular}{lccc}
\hline Subject & $\begin{array}{c}\text { Inhibition } \\
\text { Function } \\
\text { Slope }\end{array}$ & $\begin{array}{c}\text { No-Signal } \\
\text { RT Standard } \\
\text { Deviation }\end{array}$ & $\begin{array}{c}\text { Target Step } \\
\text { RT }\end{array}$ \\
\hline \multicolumn{5}{c}{$\begin{array}{c}\text { Human Step } \\
\text { C.C. }\end{array}$} & $2 / 38(5 \%)$ & $31 / 38(82 \%)$ & $0 / 38(0 \%)$ \\
L.B. & $0 / 40(0 \%)$ & $34 / 40(85 \%)$ & $0 / 40(0 \%)$ \\
S.S. & $1 / 40(3 \%)$ & $28 / 40(70 \%)$ & $0 / 40(0 \%)$ \\
\multicolumn{5}{c}{ Monkey Stop (Staircase) } \\
H & $34 / 67(51 \%)$ & $18 / 67(27 \%)$ & $0 / 67(0 \%)$ \\
N & $25 / 56(45 \%)$ & $20 / 56(36 \%)$ & $0 / 56(0 \%)$ \\
C & $28 / 34(82 \%)$ & $16 / 34(47 \%)$ & $2 / 34(6 \%)$ \\
F & $30 / 41(73 \%)$ & $18 / 41(44 \%)$ & $0 / 41(0 \%)$ \\
L & $38 / 47(81 \%)$ & $22 / 47(47 \%)$ & $0 / 47(0 \%)$ \\
T & $27 / 42(64 \%)$ & $42 / 42(100 \%)$ & $14 / 42(33 \%)$ \\
\hline
\end{tabular}

Table 3

Numbers and Proportions of Nonstaircased Sessions for Each Subject in Which a Transformation to Remove Response Time (RT) Fluctuations Significantly Affected Indicated Values at a $p<.05$ Level

\begin{tabular}{|c|c|c|c|}
\hline Subject & $\begin{array}{l}\text { Inhibition } \\
\text { Function } \\
\text { Slope }\end{array}$ & $\begin{array}{c}\text { No-Signal RT } \\
\text { Standard } \\
\text { Deviation }\end{array}$ & $\begin{array}{c}\text { Stop } \\
\text { Signal } \\
\text { RT }\end{array}$ \\
\hline A & $25 / 89(28 \%)$ & $31 / 89(35 \%)$ & $1 / 89(1 \%)$ \\
\hline $\mathrm{C}$ & $2 / 17(12 \%)$ & $6 / 17(35 \%)$ & $0 / 17(0 \%)$ \\
\hline $\mathrm{F}$ & $12 / 24(50 \%)$ & $13 / 24(54 \%)$ & $1 / 24(4 \%)$ \\
\hline $\mathrm{N}$ & $43 / 213(20 \%)$ & $106 / 213(50 \%)$ & $0 / 213(0 \%)$ \\
\hline
\end{tabular}

each subject are indicated, and the horizontal dotted lines denote the grand mean no-signal RT for each subject for comparison. We restricted this analysis to three-trial sequences for noncanceled and canceled trial types to those triplets in which the $n-1$ and $n+1$ trial were both nosignal trials. For the no-signal trial type, we just required that the $n-1$ trial type was a no-signal trial. As was shown previously, RTs appear longer following canceled trials as opposed to noncanceled trials (e.g., Emeric et al., 2007). The present analysis shows that RT was also longer before noncanceled trials. Appendix C demonstrates mathematically that these differences in RT with trial history are a simple consequence of fluctuations in RT. The proof relies on the fact that for a given SSD, more canceled trials will tend to occur when the subject is responding slowly, whereas more noncanceled trials will tend to occur when the subject is responding quickly. Thus, the incidence of successful or unsuccessful inhibition varies with RT fluctuations, which is manifest in the RTs of trials before and after the alternative responses.

To determine whether the variation of RT after different types of trials exceeds what can be explained by nonindependence and nonstationarity of RT, we performed a pair of planned interaction contrasts of RT for each combination of species and task in a $3 \times 2$ design with trial type (no signal, canceled/compensated, noncanceled/noncompensated) and trial sequence (RT before or after the target trial type) as within-subjects factors. An interaction in this design will reveal trial history effects independent of the effects of RT fluctuations. A repeated measures omnibus ANOVA using Greenhouse-Geisser adjusted degrees of freedom revealed a significant interaction of trial type and sequence in the stop task for both humans $[F(1.3,5.2)=11.7]$ and monkeys $[F(1.5,6.0)=7.1]$, but not in the step task [humans, $F(2.0,4.0)=1.7$; monkeys, $F(2.0,6.0)=2.5]$.

We investigated the omnibus interaction in the stop task further. To determine whether the magnitude of RT changes following canceled trials differs from that following noncanceled trials, we performed a paired $t$ test of the differences in RTs before and after canceled trials with the differences in RTs before and after noncanceled trials. No significant difference was found for either humans $[t(4)=-0.5]$ or monkeys $[t(4)=0.8]$. To investigate whether subjects slow their responses following canceled or noncanceled stop signal trials, relative

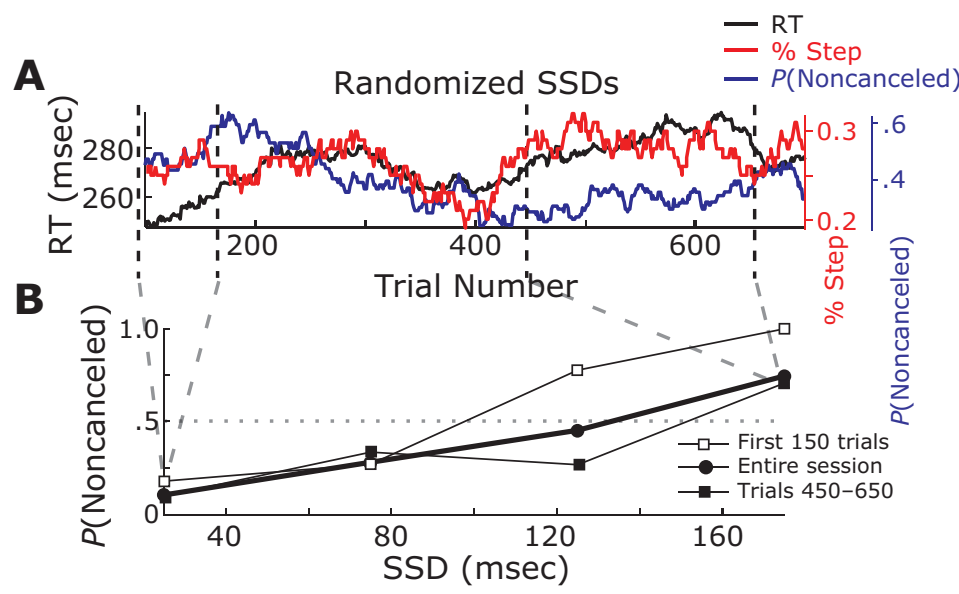

Figure 6. Effects of nonindependent and nonstationary response times (RTs) on inhibition functions when the stop signal delay (SSD) is not adaptively staircased. The conventions are the same as in Figure 5. 


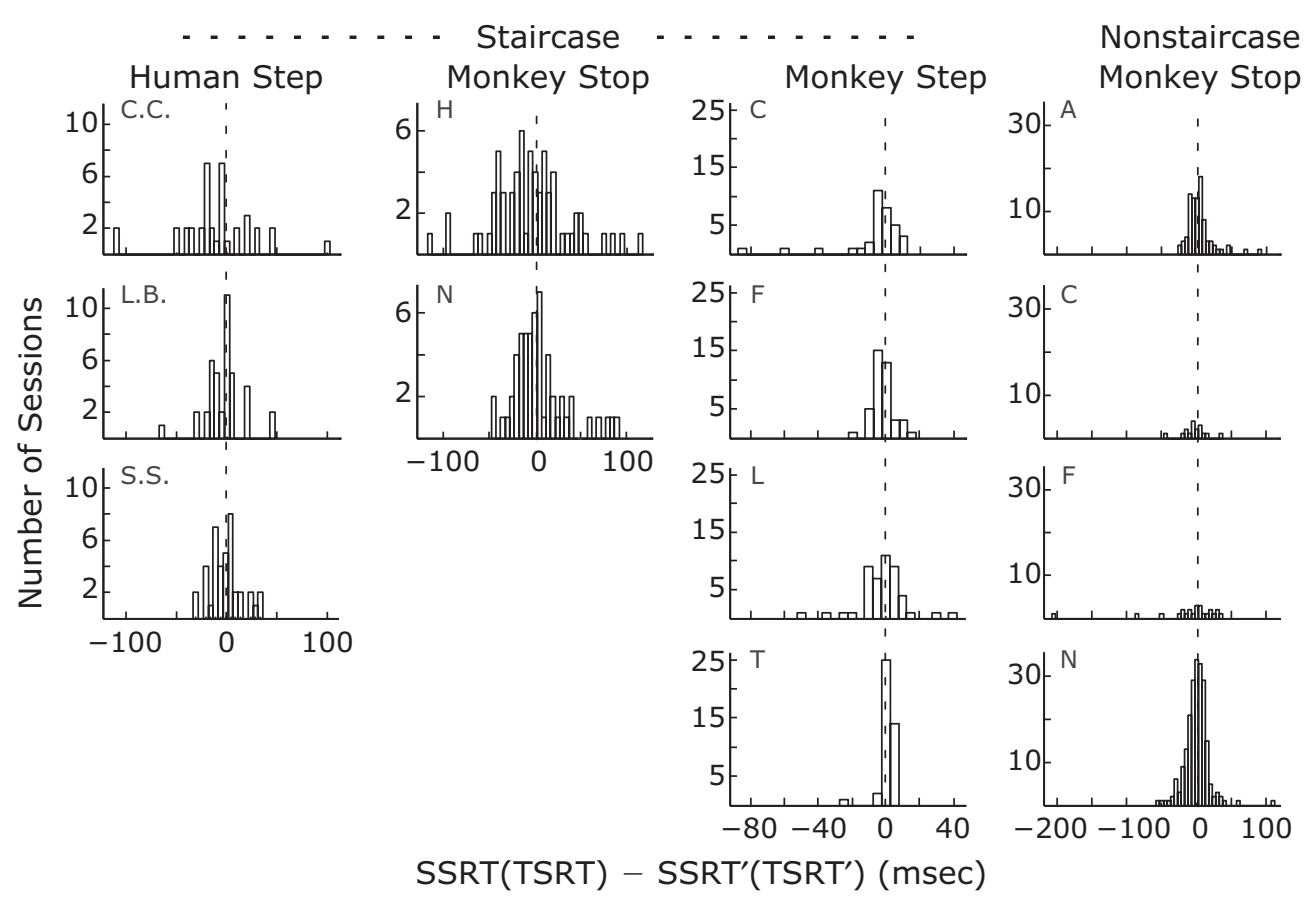

Figure 7. Effects of nonindependent and nonstationary response times (RTs) on estimations of stop signal response time (SSRT) or target step response time (TSRT). Each panel shows histograms of the original SSRT or TSRT values minus the transformed value (SSRT' or TSRT') for each session for a given subject, with plots arranged by species, task, and staircase condition, as indicated.

to responses following a no-signal trial, we performed a paired $t$ test of the differences in RTs of consecutive no-signal trials compared with the average difference in RTs before and after canceled or noncanceled trials. RTs were significantly shorter following a no-signal trial, as compared with following a canceled or a noncanceled trial [humans, $-21.7 \mathrm{msec}, t(4)=-11.0$; monkeys, $-6.0 \mathrm{msec}, t(4)=-3.6]$. Taken together, these results show that both humans and monkeys are slower following a stop trial, as opposed to following a no-signal trial, but that the amount of slowing is comparable between canceled and noncanceled trials. In the step task, generalized slowing following step trials was not observed.

\section{DISCUSSION}

We have shown that RTs in humans and monkeys performing the saccade stop signal and search step tasks are nonindependent and nonstationary across trials. However, we have shown empirically and mathematically that methods used to estimate SSRT (TSRT) are robust to such RT fluctuations. Nevertheless, nonindependence and nonstationarity of RT can result in an underestimation of the slope of the inhibition (compensation) function across a session. We have also shown how nonindependence and nonstationarity of RT influence analyses of trial history. We propose that examining the RTs on trials both before and after a trial of interest can help account for changes in RT due only to nonindependence and nonstationarity. We found that RT was relatively longer both before and after canceled stop signal trials than before and after noncan- celed trials, and that RT was not specifically longer after canceled than after noncanceled trials.

\section{Nonstationarity of the Stop Process}

We have shown that no-signal RTs in the saccade stop signal and target step tasks are nonindependent and nonstationary. Within the race model, this would be modeled as a go process that slowly varies throughout a session and that is subject to trial-to-trial correlations. The stop process also could vary throughout a session (see Appendix B). However, because measurement of the SSRT (TSRT) requires 100 or more trials to produce a stable estimate, it is difficult to demonstrate the nonstationarity of SSRT (TSRT). Thus, we have not explicitly shown the nonstationarity of the stop process.

\section{Race Model Implications}

An important contribution of this work has been to show that the calculations of SSRT (TSRT) are largely unaffected by RT fluctuations. Calculations using the mean difference method will yield a time-weighted average of the SSRT (TSRT) over the session. Validating the measurement of SSRT (TSRT) is important because of its utility as a measure of impulsivity in clinical and developmental studies (e.g., Schachar et al., 2004).

Although fluctuations in RT do not affect SSRT or TSRT calculations, we did demonstrate that it results in shallower inhibition functions. Because the slope of the inhibition function can be used to derive a measure of the variability of SSRT (Band, van der Molen, \& Logan, 2003; Colonius, 1990; Logan \& Cowan, 1984), these new results 

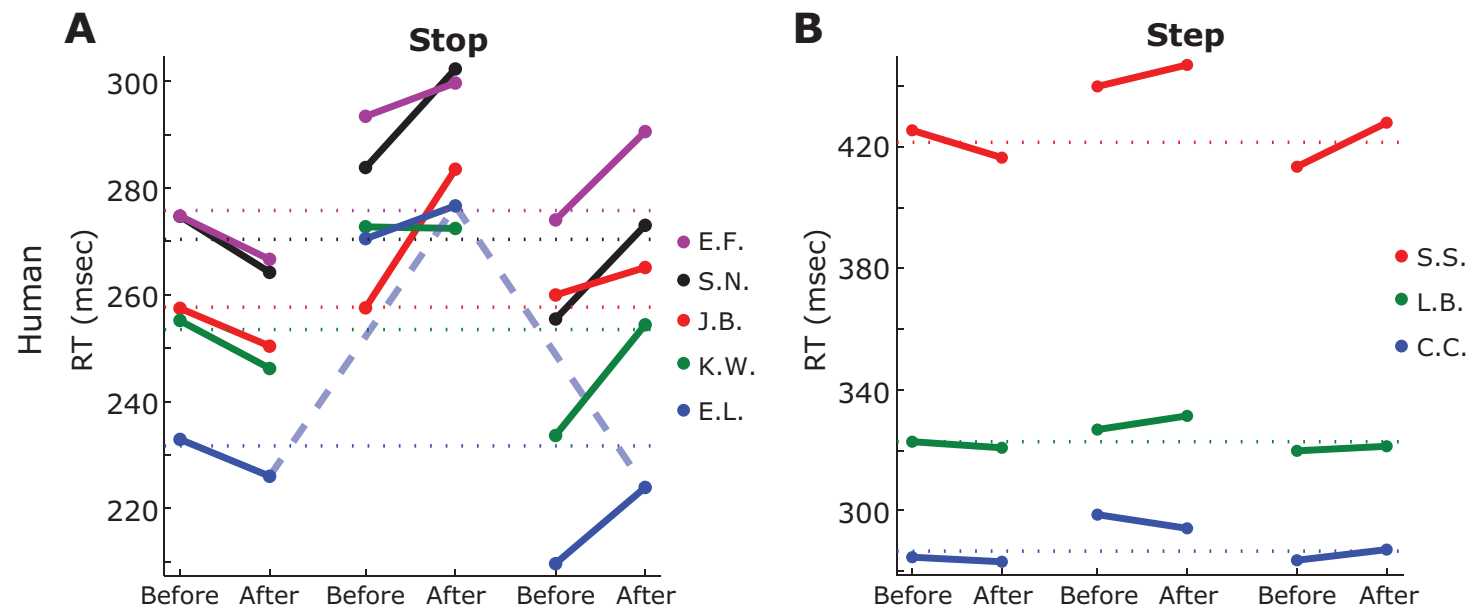

C

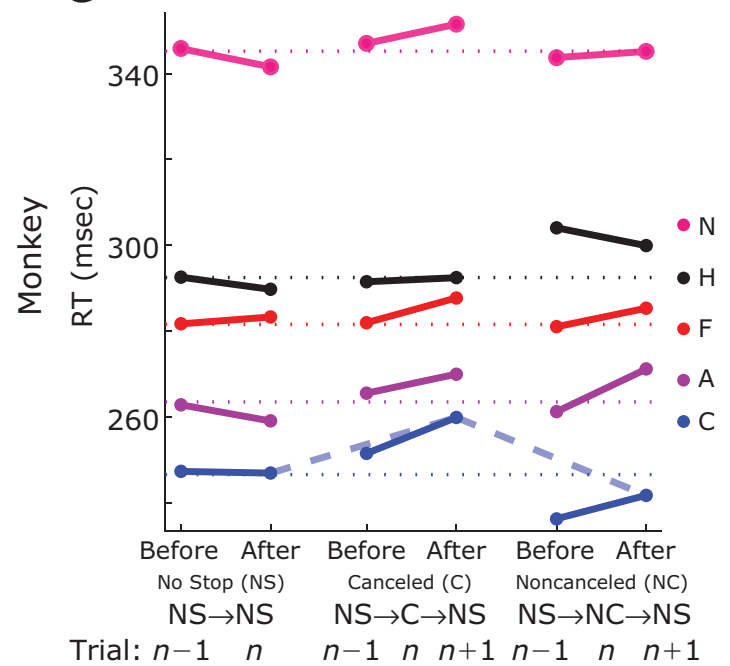

D

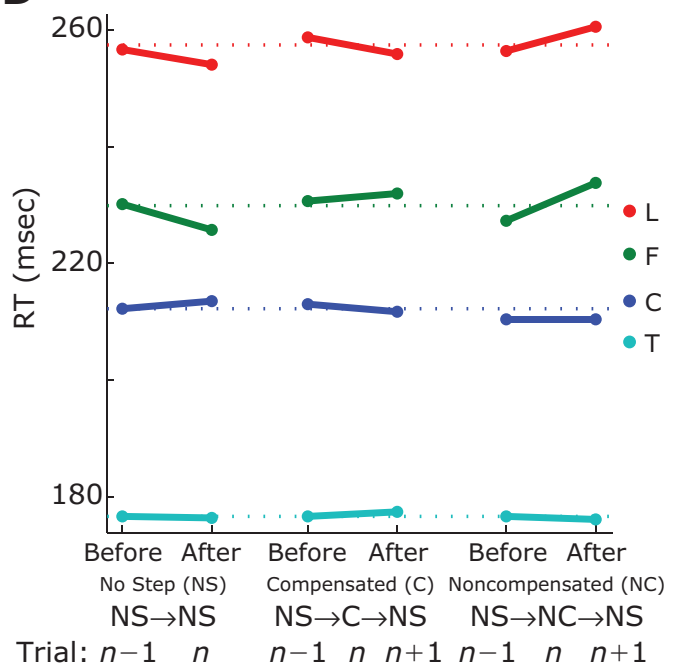

Figure 8. Trial triplet analysis. As indicated in the schematics below panels $C$ and $D$, the data included here are taken from occurrences of sequences of (from left to right) two consecutive no-signal trials, a canceled (compensated) trial with a no-signal trial before and after it, and a noncanceled (noncompensated) trial with a no-signal trial before and after it during the stop (step) task. The mean response time (RT) on the no-signal trials from each sequence are plotted as indicated for each subject, with colors corresponding to each subject as indicated beside each plot. The horizontal dotted lines show the overall mean no-signal RT for each subject. The thick dashed lines in panels A and C show, for 1 human subject and 1 monkey, the comparison that was made in Emeric et al. (2007) with these data. Panels A and C show data for human subjects and monkeys, respectively, performing the stop task, and panels B and D show data for human subjects and monkeys, respectively, performing the step task. For each condition, the monkey data in panels C and D plot the mean of all the session mean values for each subject, and the human data in panels $A$ and $B$ plot the aggregate mean value for all the trials pooled from all the sessions.

suggest that fluctuations in RT can result in an overestimation of the variability of SSRT.

\section{Choice of Staircased Versus Randomized SSDs}

A subject of methodological interest for investigators using stopping or stepping tasks is whether to adjust the SSD (TSD) by a staircase procedure or randomly select from preset values. RT fluctuations occur in both staircased and nonstaircased sessions, but as we have shown, fluctuating RTs manifest some of their effects differently, depending on how the SSDs are selected. For example, whether SSDs are staircased or randomized will affect how strongly RT fluctuations impact the trial history analyses. As RT varies during a session, a greater concentration of noncanceled trials will occur when a subject is responding quickly, whereas a greater concentration of canceled trials will occur when a subject is responding slowly. This imbalance will be particularly prevalent when the SSDs are randomized. When the staircase procedure is used, however, the proportion of canceled stop trials will be stabilized over global RT fluctuations, although there will still be some remaining bias because of local fluctuations in RT. All of the human and most of the monkey stopping data analyzed here and in Emeric et al. (2007) used randomized SSDs, which likely contributed to the magnitude of the RT shifts between the trials before canceled and noncanceled trials.

In addition, when staircasing is used, SSDs fluctuate in response to RT fluctuations, which creates a correlation of 
RT with SSD. We have found this correlation to be significant in most individual staircased sessions across species and tasks. One practical consequence of this is that this correlation should be taken into account when noncanceled RTs are compared with no-signal RTs. To verify that behavior conforms to the race model, investigators often compare the distribution of noncanceled RTs at each SSD with the distribution of no-signal RTs over the whole session. If, at any SSD, the noncanceled distribution exceeds the no-signal distribution, this is seen as a violation of the independence premise of the race model. However, when staircasing is used, this may not be a valid comparison, because this correlation dictates that the overall no-signal RT distribution will be different from the RT distribution at each SSD (TSD), particularly at the highest and lowest SSDs (TSDs). Instead, it may be preferable to compare the noncanceled RT distribution at each SSD (TSD) with the RT distribution on no-signal trials when that same SSD (TSD) was expected.

In contrast to when staircased, when randomized SSD (TSD) is used, the values remain constant despite the RT fluctuations. Instead of a positive correlation between SSD (TSD) and RT, there is a negative correlation between the probability of responding on a stop (step) trial and RT, as one would expect from the race model.

\section{Stopping Trial History}

We have shown that inferences about the effect that events on trial $n$ have on performance on trial $n+1$ must control for the nonindependence and nonstationarity of RT. We suggest using the no-signal trials on trial $n-1$ as a simple control comparison (since events on trial $n$ cannot affect events on prior trial $n-1)$. Of course, one could argue that trial $n-1$ is preceded by other trial types that would influence its RT, which, in turn, are influenced by other preceding trials, and so on. However, in practice, the numbers of equivalent trials preceding $n-1$ or following $n+1$ and beyond in these tasks are too few for sufficient statistical power.

Cabel et al. (2000) and Emeric et al. (2007) found greater lengthening of RTs following canceled than following noncanceled trials. Emeric et al. (2007) also reported no systematic lengthening of RTs following noncanceled trials. This apparent lack of posterror slowing following a missed stop signal was surprising, given the apparent ubiquity of posterror slowing in a range of tasks (e.g., Hajcak, McDonald, \& Simons, 2003; Rabbit, 1966). However, when we accounted for RT nonindependence and nonstationarity by examining trial $n-1$, we found that subjects are indeed slower to respond on trials following noncanceled (error) trials. Of note, however, subjects lengthened their RTs to the same degree following canceled (correct) trials. Thus, the present results indicate a general lengthening of RT following any stop signal trial. Clearly, accounting for RT nonindependence and nonstationarity has important implications for understanding alternative mechanisms whereby trial history affects performance (e.g., Botvinick, Braver, Barch, Carter, \& Cohen, 2001; Schall \& Boucher, 2007; Verbruggen, Logan, et al., 2008).

\section{AUTHOR NOTE}

This work was supported by Grants NIH R01-MH55806, NIH R01EY08890, NIH F32-EY016679, AFOSR FA 9550-07-1-0192, NSF SBE-0542013, NIH P30-EY08126, and NIH P30-HD015052; by Grant SBE-0542013 from the Temporal Dynamics of Learning Center; by an NSF Science of Learning Center grant; and by Robin and Richard Patton through the E. Bronson Ingram Chair of Neuroscience. We thank Doug Hanes, Veit Stuphorn, Alex Gotler, Adi Murthy, and Kirk Thompson for their efforts in the original data collection; Corrie Camalier and Erik Emeric for assistance working with the data sets as well as for the original data collection; Courtney Segovis for assistance with the manuscript; and Pierre Pouget and Richard Heitz for helpful discussions. Correspondence concerning this article should be addressed to J. D. Schall, Vanderbilt Vision Research Center, Department of Psychology, Vanderbilt University, PMB 407817, 2301 Vanderbilt Place, Nashville, TN 372407817 (e-mail: jeffrey.d.schall@vanderbilt.edu).

\section{REFERENCES}

Band, G. P., van der Molen, M. W., \& Logan, G. D. (2003). Horserace model simulations of the stop-signal procedure. Acta Psychologica, 112, 105-142.

BECKER, W., \& JÜRGENS, R. (1979). An analysis of the saccadic system by means of double step stimuli. Vision Research, 19, 967-983.

Botvinick, M. M., Braver, T. S., Barch, D. M., Carter, C. S., \& Cohen, J. D. (2001). Conflict monitoring and cognitive control. Psychological Review, 108, 624-652.

Boucher, L., Palmeri, T. J., Logan, G. D., \& Schall, J. D. (2007). Inhibitory control in mind and brain: An interactive race model of countermanding saccades. Psychological Review, 114, 376-397.

Boucher, L., Stuphorn, V., Logan, G. D., Schall, J. D., \& Palmeri, T. J. (2007). Stopping eye and hand movements: Are the processes independent? Perception \& Psychophysics, 69, 785-801.

Broadbent, D. E. (1971). Decision and stress. London: Academic Press.

Cabel, D. W., Armstrong, I. T., Reingold, E., \& Munoz, D. P. (2000). Control of saccade initiation in a countermanding task using visual and auditory stop signals. Experimental Brain Research, 133, 431-441.

Camalier, C. R., Gotler, A., Murthy, A., Thompson, K. G., Logan, G. D., Palmeri, T. J., \& Schall, J. D. (2007). Dynamics of saccade target selection: Race model analysis of double step and search step saccade production in human and macaque. Vision Research, 47, 2187-2211.

Colonius, H. (1990). A note on the stop-signal paradigm or how to observe the unobservable. Psychological Review, 97, 309-312.

Emeric, E. E., Brown, J. W., Boucher, L., Carpenter, R. H., Hanes, D. P., HARRIS, R., ET AL. (2007). Influence of history on saccade countermanding performance in humans and macaque monkeys. Vision Research, 47, 35-49.

Fecteau, J. H., \& Munoz, D. P. (2003). Exploring the consequences of the previous trial. Nature Reviews Neuroscience, 4, 435-443.

Freeman, G. L. (1933). The facilitative and inhibitory effects of muscular tension upon performance. American Journal of Psychology, 26, 602-608

Gilden, D. L. (2001). Cognitive emissions of $1 / f$ noise. Psychological Review, 108, 33-56.

Hajcak, G., McDonald, N., \& Simons, R. F. (2003). To err is autonomic: Error-related brain potentials, ANS activity and post-error compensatory behavior. Psychophysiology, 40, 895-903.

Hanes, D. P., Patterson, W. F., \& Schall, J. D. (1998). The role of frontal eye field in countermanding saccades: Visual, movement and fixation activity. Journal of Neurophysiology, 79, 817-834.

Hanes, D. P., \& Schall, J. D. (1995). Countermanding saccades in macaque. Visual Neuroscience, 12, 929-937.

JARvis, M. R., \& Mitra, P. P. (2001). Sampling properties of the spectrum and coherency of sequences of action potentials. Neural Computation, 13, 717-749.

Kornylo, K., Dill, N., Saenz, M., \& Krauzlis, R. J. (2003). Canceling of pursuit and saccadic eye movements in human and monkeys. Journal of Neurophysiology, 89, 2984-2999. 
Li, C. S., Krystal, J. H., \& Mathalon, D. H. (2005). Fore-period effect and stop-signal reaction time. Experimental Brain Research, 167, 305-309.

LogAN, G. D. (1985). Skill and automaticity: Relations, implications and future directions. Canadian Journal of Psychology, 39, 367386.

LogAN, G. D. (1994). On the ability to inhibit thought and action: A user's guide to the stop signal paradigm. In D. Dagenbach \& T. H. Carr (Eds.), Inhibitory processes in attention, memory, and language (pp. 189-239). San Diego: Academic Press.

LogAn, G. D., \& CowAN, W. B. (1984). On the ability to inhibit simple and choice reaction time responses: A theory of an act of control. Psychological Review, 91, 295-327.

Logan, G. D., Schachar, R. J., \& TANNOCK, R. (1997). Impulsivity and inhibitory control. Psychological Science, 8, 60-64.

Lomb, N. R. (1976). Least-squares frequency analysis of unequally spaced data, Astrophysical \& Space Science, 39, 447-462.

LuCE, R. D. (1986). Response times: Their role in inferring elementary mental organization. New York: Oxford University Press.

Murthy, A., Ray, S., Shorter, S. M., Priddy, E. G., Schall, J. D., \& Thompson, K. G. (2007). Frontal eye field contributions to rapid corrective saccades. Journal of Neurophysiology, 97, 1457-1469.

Murthy, A., Ray, S., Shorter, S. M., Schall, J. D., \& Thompson, K. G. (2009). Neural control of visual search by frontal eye field: Effects of unexpected target displacement on visual selection and saccade preparation. Journal of Neurophysiology, 101, 2485-2506.

Osman, A., Kornblum, S., \& Meyer, D. E. (1990). Does motor programming necessitate response execution? Journal of Experimental Psychology: Human Perception \& Performance, 16, 183-198.

Özyurt, J., Colonius, H., \& Arndt, P. A. (2003). Countermanding saccades: Evidence against independent processing of go and stop signals. Perception \& Psychophysics, 65, 420-428.

Paré, M., \& Hanes, D. P. (2003). Saccadic reaction time in the monkey: Advanced preparation of oculomotor programs is primarily responsible for express saccade occurrence. Journal of Neurophysiology, 76, 3666-3681.
Percival, D. B., \& Walden, A. T. (1993). Spectral analysis for physical applications. Cambridge: Cambridge University Press.

RaBBIT, P. M. (1966). Error correction time without external error signals. Nature, 212, 438.

Rieger, M., \& GAUGGel, S. (1999). Inhibitory after-effects in the stop signal paradigm. British Journal of Psychology, 90, 509-518.

Scargle, J. D. (1982). Studies in astronomical time series analysis: II. Statistical aspects of spectral analysis of unevenly spaced data. Astrophysical Journal, 263, 835-853.

Schachar, R. J., Chen, S., Logan, G. D., Ornstein, T. J., Crosbie, J., ICKOWICZ, A., \& PAKULAK, A. (2004). Evidence for an error monitoring deficit in attention deficit hyperactivity disorder. Journal of Abnormal Child Psychology, 32, 285-293.

Schall, J. D., \& Boucher, L. (2007). Executive control of gaze by the frontal lobes. Cognitive, Affective, \& Behavioral Neuroscience, 7, 396-412.

Verbruggen, F., \& Logan, G. D. (2008a). Long-term aftereffects of response inhibition: Memory retrieval, task goals, and cognitive control. Journal of Experimental Psychology: Human Perception \& Performance, 34, 1229-1235.

Verbruggen, F., \& Logan, G. D. (2008b). Response inhibition in the stop-signal paradigm. Trends in Cognitive Sciences, 12, 418-424.

Verbruggen, F., Logan, G. D., Liefooghe, B., \& Vandierendonck, A. (2008). Short-term aftereffects of response inhibition: Repetition priming or between-trial control adjustments. Journal of Experimental Psychology: Human Perception \& Performance, 34, 413-426.

Verbruggen, F., Schneider, D. W., \& Logan, G. D. (2008). How to stop and change a response: The role of goal activation in multitasking. Journal of Experimental Psychology: Human Perception \& Performance, 34, 1212-1228.

Wagenmakers, E.-J., Farrell, S., \& RatclifF, R. (2004). Estimation and interpretation of $1 / f^{\alpha}$ noise in human cognition. Psychonomic Bulletin \& Review, 11, 579-615.

Welford, A. T. (1968). Fundamentals of skill. London: Methuen.

Welford, A. T. (1980). Choice reaction time: Basic concepts. In A. T. Welford (Ed.), Reaction times (pp. 73-128). New York: Academic Press.

\section{APPENDIX A \\ Spectral Methods}

Here, we provide background to the spectral analyses we used to test whether our RT data display power spectra that deviate from the flat spectrum associated with an independent time series.

For a given discretely sampled time series $x[t]$ with a constant interval between samples, a direct estimate of its power spectrum can be obtained through the conventional periodogram. As is shown below, the periodogram reflects a normalized square of a correlation of the time series with sinusoidal signals at a given frequency across all time points in the sample:

$$
P_{x}[f]=\frac{1}{N_{o}}\left|\sum_{t=1}^{N_{o}} x[t] e^{-2 \pi i f t}\right|^{2}
$$

or equivalently

$$
P_{x}[f]=\frac{1}{N_{o}}\left[\left(\sum_{t=1}^{N_{o}} x[t] \cos (2 \pi f t)\right)^{2}+\left(\sum_{t=1}^{N_{o}} x[t] \sin (2 \pi f t)\right)^{2}\right],
$$

where $N_{0}$ refers to the number of time points in the sample.

Such computations are often performed on signals that are digital samples over time of some underlying temporally continuous value. Examples from electrophysiology research include a local field potential or an electroencephalogram channel. Here, we consider RT spectra analyses that are analogous to these signals, but with differences in the underlying implications. The unit of "time" in these analyses is an ultimately discrete unit of trial numbers, rather than a precise unit of time like seconds or milliseconds. We thus consider frequency in units of inverse trial numbers, rather than in Hertz. However, in this analysis, the concept of frequency may be more easily conceptualized by considering the corresponding period of a given frequency, which has units of trials. For the dependent variable of the time series, instead of volts (or indeed, microvolts), we measure RT in units of milliseconds and measure power in units of milliseconds squared. The maximum possible sampling rate is fixed at one sample per trial, which results in a Nyquist frequency limit of 0.5 inverse trials that we can meaningfully consider. 


\section{APPENDIXA (Continued)}

The spectral analysis associated with the stopping and stepping tasks we consider in this article comes with an additional caveat. We are interested in the spectrum of the go process, which can be directly measured from the RTs of no-signal trials only (Logan \& Cowan, 1984). This means that we can only include these trials' RTs in our spectral analysis, which results in a missing datum for every stop or step trial. Importantly, it would be incorrect to ignore these missing data and analyze the sequence of no-signal trials using only techniques that assume regularly spaced data samples. Instead, to accurately measure the RT power spectra, we must take into account the specific trial position of every no-signal RT. To do so, we used techniques described in Lomb (1976) and Scargle (1982) for the spectral analysis of irregularly spaced data, which we briefly review below.

Scargle (1982) presented the use of a modified periodogram:

$$
P(f)=\frac{1}{2}\left\{\frac{\left[\sum_{j} X\left(t_{j}\right) * \cos \left(2 \pi f\left(t_{j}-\tau\right)\right)\right]^{2}}{\sum_{j} \cos ^{2}\left(2 \pi f\left(t_{j}-\tau\right)\right)}+\frac{\left[\sum_{j} X\left(t_{j}\right) * \sin \left(2 \pi f\left(t_{j}-\tau\right)\right)\right]^{2}}{\sum_{j} \sin ^{2}\left(2 \pi f\left(t_{j}-\tau\right)\right)}\right\},
$$

where $\tau$ is defined as

$$
\tau=\frac{1}{2 * 2 \pi f} \tan ^{-1}\left[\frac{\sum_{j} \sin \left(2 * 2 \pi f t_{j}\right)}{\sum_{j} \cos \left(2 * 2 \pi f t_{j}\right)}\right] .
$$

Using the details described in Lomb (1976), Scargle demonstrated that this is the equivalent of a least-squares fit of sine waves to irregularly spaced data. Indeed, regularly spaced data can be seen as a special case of this more general measure, since Scargle shows that, in this case, these expressions reduce to the conventional expressions for periodograms. The effect of the parameter $\tau$ is to maintain the invariance of the estimate to a time shift in the input time series, as well as to preserve the accurate relative phases between different frequencies. For the special case of regularly spaced data, $\tau$ will equal zero. As Scargle shows, the other modifications of the periodogram serve to produce resulting estimates of spectral power that have the same statistical properties as the conventional periodogram with regularly spaced data. On the basis of this, we use statistical techniques that were described in Jarvis and Mitra (2001) and developed in Percival and Walden (1993). Specifically, a 95\% confidence interval for the population spectral power at a given frequency, $P_{\text {pop }}(f)$, given the estimated spectral power, $P(f)$, is given by

$$
\frac{v_{o} P(f)}{q_{2}} \leq P_{\text {pop }}(f) \leq \frac{v_{o} P(f)}{q_{1}},
$$

where $q_{1}$ and $q_{2}$, respectively, refer to the 2.5 th and 97.5 th percentiles of the chi-square distribution with degrees of freedom of $v_{o}$. In our case, the degrees of freedom are equal to twice the number of sessions averaged across. Before averaging across sessions, we also averaged spectral estimates in adjacent frequency bins within each session, but we did not add these estimates to our total degrees of freedom, since some of these adjacent estimates would not be totally independent.

\section{APPENDIX B \\ Effect of Nonindependence and Nonstationarity on SSRT Calculation With the Mean-Difference Method}

Here, we will show that one common measurement of SSRT is robust to the nonindependent and nonstationary fluctuations that we have shown to exist in RT data. This holds provided that the proportion of stop trials presented to the subject remains constant through the RT changes. In particular, here, we consider SSRT calculation using the mean-difference method (see Logan \& Cowan, 1984).

For simplification, in the following formulation, we consider the stop and go processes in discrete epochs of arbitrary numbers of trials, with each process being stationary within an epoch. In the extreme case, these epochs of stationarity can be as short as one trial, which makes no assumption of stationarity between any trials. The logic of the formulation would remain the same in this case, with two minor differences. The epoch means we describe would, instead, be considered to be the expected value of a single trial's RT, rather than the mean RT across an epoch, and the value of $w_{i}$ for all $i$ s could be simplified to $1 / N$, where $N$ is the number of trials in the session.

Using conventions from Logan and Cowan (1984), let $f(t)$ denote the probability density function of the distribution of go process finish times as measured by the RTs on no-signal trials over the entire session. Let $w_{i}$ denote the proportion of the total number of no-signal trials over the entire session that is contained within epoch $i$, and let $f_{i}(t)$ denote the distribution of go process finish times within that epoch. Thus, we define that

$$
f(t)=\Sigma_{i} w_{i} f_{i}(t)
$$

The mean go process finish time $\bar{T}$ is thus

$$
\bar{T}=\int_{-\infty}^{\infty} t f(t) d t=\int_{-\infty}^{\infty} t\left[\sum_{i} w_{i} f_{i}(t)\right] d t=\sum_{i} w_{i} \int_{-\infty}^{\infty} t f_{i}(t) d t=\sum_{i} w_{i} \bar{T}_{i},
$$




\section{APPENDIX B (Continued)}

where $\bar{T}_{i}$ is the mean go process finish time for epoch $i$.

As was described in Logan and Cowan (1984), the inhibition function, which is the probability of not canceling an initial saccade on a stop trial with a given SSD, can be treated as the cumulative distribution of some random function $T_{d}$. Let $T_{d}$ be distributed as the function $\phi\left(t_{d}\right)$ such that

$$
\phi\left(t_{d}\right)=\frac{d P_{r}\left(t_{d}\right)}{d t}
$$

where $P_{r}\left(t_{d}\right)$ is the probability of responding on a stop trial with an SSD of $t_{d}$. For simplicity, we will assume that the duration of the ballistic component of the movement is zero, although that can be added to the model for completeness without affecting these results. Let $f_{s i}(t)$ denote the distribution of stop process finish times within each epoch. Thus for epoch $i$, the distribution of $T_{d}$ is given by

$$
\phi_{i}\left(t_{d}\right)=\int_{-\infty}^{\infty} f_{s i}(u) f_{i}\left(u+t_{d}\right) d u
$$

For the entire session,

$$
\phi\left(t_{d}\right)=\sum_{i} w_{i} \phi_{i}\left(t_{d}\right)=\sum_{i} w_{i} \int_{-\infty}^{\infty} f_{s i}(u) f_{i}\left(u+t_{d}\right) d u .
$$

Importantly, if the ratio of stop trials is identical in each epoch, then each $w_{i}$ here will correspond to the $w_{i}$ for the no-signal distribution in Equation B1 as the weight of each epoch's contribution to the entire session's inhibition function, and the no-signal distribution will be the same.

We can then calculate the mean of this distribution as

$$
\bar{T}_{d}=\int_{-\infty}^{\infty} t_{d} \phi\left(t_{d}\right) d t_{d}=\sum_{i} w_{i} \int_{-\infty}^{\infty} t_{d} \int_{-\infty}^{\infty} f_{s i}(u) f_{i}\left(u+t_{d}\right) d u d t_{d} .
$$

With the observation that

$$
\int_{-\infty}^{\infty} t_{d} \int_{-\infty}^{\infty} f_{s i}(u) f_{i}\left(u+t_{d}\right) d u d t_{d}=\bar{T}_{i}-\bar{T}_{s i}
$$

where $\bar{T}_{s i}$ is the mean stop process finish time for epoch $i$, Equation B6 can be rewritten as

$$
\bar{T}_{d}=\sum_{i} w_{i}\left(\bar{T}_{i}-\bar{T}_{s i}\right)=\sum_{i} w_{i} \bar{T}_{i}-\sum_{i} w_{i} \bar{T}_{s i}
$$

The difference-of-means method presented in Logan and Cowan (1984) suggests that one method to estimate SSRT for a given data set is to subtract the mean of the inhibition function from the mean of the no-signal RT distribution for the entire session. Applying Equations B2 and B7, we see that if the distributions of the go and stop process vary across epochs within a session, this measurement would still amount to a weighted average of the SSRT in each epoch:

$$
\bar{T}-\bar{T}_{d}=\sum_{i} w_{i} \bar{T}_{s i}
$$

This would be the measurement of interest in most cases even if the SSRT distribution does vary within a session, and so this measurement of SSRT is robust to fluctuations in RT and SSRT during a session, given that the assumptions in the analysis hold.

One assumption that would not hold strictly true is that the fraction of stop trials is constant in every epoch simply due to randomness in the determination of stop trials and the timing of changes in RT. If this happens, it would bias the measurement of SSRT to some extent. For example, if, during a given epoch in which the subject is responding more quickly than normal, the subject was also presented with more stop trials than normal, that epoch would have a disproportionate effect in determining the inhibition function for the overall session than it would in determining the no-signal RT distribution for the entire session. This would shift the inhibition function earlier in time, so that the no-signal RT distribution and resulting measurements of SSRT for the entire session would overestimate SSRT.

It is also worth noting that a change in the proportion of stop trials arising by chance can induce changes in RT (Emeric et al., 2007). This would suggest that these spontaneously occurring epochs could serve to bias the calculation of SSRT, resulting in some degree of underestimation of SSRT, following the logic described above. For this reason, it may be wise for experimenters to follow the common practice of pseudorandomizing the presentation of stop trials to prevent long stretches of too few or too many stop trials locally to mitigate this effect.

This would also suggest that a session with rapidly fluctuating RTs would result in more noise being added to the estimation of SSRT but that measurements made from longer sessions should be more robust to the effects of this noise. 


\section{APPENDIX C}

\section{Effect of RT Nonindependence and Nonstationarity During Stopping or Stepping Tasks} on Measurements of RTs Before and After Canceled and Noncanceled Trials

Here, we will show that fluctuations in RT will cause the mean RT on no-signal trials before and after canceled trials to be higher than the mean RT on no-signal trials before and after noncanceled trials even when the presence of a canceled or a noncanceled trial does not have any effect on the distribution of no-signal RTs on any trial. The proof below explicitly shows this for trials following a canceled or a noncanceled trial, but the same logic applies to trials preceding a canceled or a noncanceled trial.

For the sake of simplicity, we will consider the case in which an experimental session consists of two epochs of equal size and the SSDs are randomized such that each SSD is presented with the same probability in the two epochs. In Epoch 1, suppose that the cumulative distribution function of the go process finish times are given by some function $F(t)$, with an expected value of $T$, and that the finish time distribution in Epoch 2 is the same but slowed by a constant amount, $t_{\text {slow }}$. Thus, in Epoch 2 the cumulative distribution function of go finish times is given by $F\left(t-t_{\text {slow }}\right)$ with an expected value of $\bar{T}+t_{\text {slow }}$.

Let $\mathrm{RT}_{\mathrm{NC} 1 i}$ and $\mathrm{RT}_{\mathrm{NC} 2 i}$ denote the $i$ th $\mathrm{RT}$ on a no-signal trial following a noncanceled trial in the first and second epochs, respectively, and likewise let $\mathrm{RT}_{\mathrm{C} 1 i}$ and $\mathrm{RT}_{\mathrm{C} 2 i}$ denote the ith $\mathrm{RT}$ on a no-signal trial following a canceled trial in the first and second epochs, respectively. Let the mean RT on no-signal trials following noncanceled and canceled trials be given by $\bar{T}_{\mathrm{NC}}$ and $\bar{T}_{\mathrm{C}}$, respectively. Then,

and

$$
\bar{T}_{\mathrm{NC}}=\frac{1}{N_{1 \mathrm{NC}}+N_{2 \mathrm{NC}}} *\left(\sum_{i}^{N_{1 \mathrm{NC}}} \mathrm{RT}_{\mathrm{NC} 1 i}+\sum_{i}^{N_{2 \mathrm{NC}}} \mathrm{RT}_{\mathrm{NC} 2 i}\right)
$$

$$
\bar{T}_{\mathrm{C}}=\frac{1}{N_{1 \mathrm{C}}+N_{2 \mathrm{C}}} *\left(\sum_{i}^{N_{1 \mathrm{C}}} \mathrm{RT}_{\mathrm{C} 1 i}+\sum_{i}^{N_{2 \mathrm{C}}} \mathrm{RT}_{\mathrm{C} 2 i}\right),
$$

where $N_{1 \mathrm{NC}}$ and $N_{2 \mathrm{NC}}$ are the total numbers of no-signal trials following noncanceled trials in the first and second epochs, respectively, and $N_{1 \mathrm{C}}$ and $N_{2 \mathrm{C}}$ are the total numbers of no-signal trials following canceled trials in the first and second epochs, respectively. Focusing on noncanceled trials, because $\mathrm{RT}_{\mathrm{NC} 1 i}$ and $\mathrm{RT}_{\mathrm{NC} 2 i}$ are determined from independent identically distributed samples of the go process's finish time distribution, this means that

$$
E\left(\bar{T}_{\mathrm{NC}}\right)=\frac{E\left(N_{1 \mathrm{NC}}\right) * \bar{T}+E\left(N_{2 \mathrm{NC}}\right) *\left(\bar{T}+t_{\text {slow }}\right)}{E\left(N_{1 \mathrm{NC}}\right)+E\left(N_{2 \mathrm{NC}}\right)} .
$$

For the sake of simplicity, let SSRT be the same constant value between epochs, $\bar{T}_{s}$. Let the variable $N_{t d j}$ denote the number of stop trials in an epoch at a given SSD value $t_{d j}$. By the logic of the race model,

and

$$
E\left(N_{1 \mathrm{NC}}\right)=\sum_{j} F\left(\bar{T}_{s}+t_{d j}\right) * E\left(N_{t d j}\right)
$$

$$
E\left(N_{2 \mathrm{NC}}\right)=\sum_{j} F\left(\bar{T}_{s}+t_{d j}-t_{\text {slow }}\right) * E\left(N_{t d j}\right)
$$

Note that when a staircase procedure is not used and the SSD is randomized, as we consider here, the expectation of $N_{t d j}$ is the same for both epochs. Similarly, for canceled trials,

$$
E\left(N_{1 \mathrm{C}}\right)=\sum_{j}\left[1-F\left(\bar{T}_{s}+t_{d j}\right)\right] * E\left(N_{t d j}\right)
$$

and

$$
E\left(N_{2 \mathrm{C}}\right)=\sum_{j}\left[1-F\left(\bar{T}_{s}+t_{d j}-t_{\text {slow }}\right)\right] * E\left(N_{t d j}\right) .
$$

The function $F$ is a monotonically increasing cumulative distribution, so, for any $t_{d j}$,

$$
F\left(\bar{T}_{s}+t_{d j}-t_{\text {slow }}\right) \leq F\left(\bar{T}_{s}+t_{d j}\right),
$$

where the limit of equality is reached only if the value of does not change anywhere in the range from $\bar{T}_{s}+t_{d j}-$ $t_{\text {slow }}$ to $\bar{T}_{s}+t_{d j}$. Thus,

and

$$
E\left(N_{1 \mathrm{NC}}\right) \geq E\left(N_{2 \mathrm{NC}}\right)
$$

$$
E\left(n_{1 \mathrm{C}}\right) \leq E\left(n_{2 \mathrm{C}}\right)
$$

Equation 2 can be rewritten as

$$
E\left(\bar{T}_{N C}\right)=\left(1-w_{2 \mathrm{NC}}\right) * \bar{T}+w_{2 \mathrm{NC}} *\left(\bar{T}+t_{\text {slow }}\right)=\bar{T}+w_{2 \mathrm{NC}} * t_{\text {slow }},
$$




\section{APPENDIX C (Continued)}

where

$$
w_{2 \mathrm{NC}}=\frac{E\left(N_{2 \mathrm{NC}}\right)}{E\left(N_{1 \mathrm{NC}}\right)+E\left(N_{2 \mathrm{NC}}\right)} .
$$

Similarly, it can be shown that

$$
E\left(\bar{T}_{\mathrm{C}}\right)=\bar{T}+w_{2 \mathrm{C}} * t_{\text {slow }},
$$

where

$$
w_{2 \mathrm{C}}=\frac{E\left(N_{2 \mathrm{C}}\right)}{E\left(N_{1 \mathrm{C}}\right)+E\left(N_{2 \mathrm{C}}\right)} .
$$

Using Equations $\mathrm{C} 6$ and $\mathrm{C} 7$, we see that $w_{2 \mathrm{C}} \geq 0.5 \geq w_{2 \mathrm{NC}}$, and thus,

$$
E\left(\bar{T}_{\mathrm{C}}\right) \geq E\left(\bar{T}_{\mathrm{NC}}\right)
$$

even though there is no direct effect of any canceled or noncanceled trial slowing the response on the following trial.

(Manuscript received October 30, 2009;

revision accepted for publication May 4, 2010.) 